\title{
Mechanische Abtrennung biogener Reststoffe aus Restabfall für die Co-Vergärung in Faultürmen kommunaler Kläranlagen
}

\author{
Michael Meirer · Wolfgang Müller · Anke Bockreis
}

Online publiziert: 10. Juli 2017

(C) Der/die Autor(en) 2017. Dieser Artikel ist eine Open-Access-Publikation.

Zusammenfassung Die Co-Vergärung getrennt gesammelter Bioabfälle gemeinsam mit kommunalen Klärschlämmen führt zu einer Unterbrechung des Nährstoffkreislaufs, wenn Klärschlämme nicht mehr als Dünger verwendet und zukünftig sogar verbrannt werden müssen.

In diesem Artikel wird der Ansatz beschrieben, die im Restabfall enthaltenen biogenen Reststoffe als Substitut für derzeit eingesetzte Co-Substrate zu nutzen. Dazu wurden Restabfälle in einer 1 . Phase charakterisiert und darauf aufbauend in einer 2. Phase mechanisch aufbereitet, um die Abtrennung eines möglichst störstofffreien Co-Substrats zu erzielen.

Enthielt der untersuchte Restabfall aus Tirol knapp $20 \%$ an Organik, lag dieser Wert in der Fraktion $<40 \mathrm{~mm}$ bereits bei $47 \%$ und in der Fraktion $<10 \mathrm{~mm}$ sogar bei $67 \%$. Nach der Aufbereitung konnten Substrate mit bis $\mathrm{zu} 80 \%$ an organischer Trockensubstanz und einem Biogaspotenzial von ca. $760 \mathrm{~m}^{3}$ pro $\mathrm{Mg}$ organischer Trockensubstanz erzeugt werden. Eine großtechnische Umsetzung wird derzeit in einer 3. Phase vorbereitet, um Schwankungen des heterogenen Restabfalls ausgleichen zu können.
Mechanical separation of organic residues in residual wastes for co-digestion in anaerobic digesters at waste water treatment plants

Abstract The co-fermentation of separately collected biowaste alongside municipal sewage sludge leads to an interruption of the nutrient cycle if sewage sludge is consequently no longer used as a fertilizer and has to be burned in the future.

This work describes the approach to utilizing the biogenic residues contained in the residual waste as a substitute for currently used co-substrates. To this end, residual waste was characterized in a first phase and then mechanically treated in a second phase in order to achieve the separation of a co-substrate which is free from any possible contamination.

The total residual waste from Tyrol contained almost $20 \%$ of organic matter, the fraction $<40 \mathrm{~mm}$ already $47 \%$ and the fraction $<10 \mathrm{~mm} 67 \%$. After processing, substrates with up to $80 \%$ volatile solids and a biogas potential of approximately $760 \mathrm{~m}^{3}$ per $\mathrm{Mg}$ volatile solids could be produced. A large-scale technical implementation is currently being prepared in a third phase in order to compensate fluctuations in the heterogeneous residual waste.

\section{Einleitung}

\subsection{Stand der Technik Co-Vergärung}

Die Co-Vergärung auf kommunalen Kläranlagen ermöglicht es, freie Faulraumkapazitäten zu nutzen und damit die Wirtschaftlichkeit einer Abwasserreinigungsanlage (ARA) zu verbessern. Durch die Zugabe von Co-Substraten zum Primär- und Überschussschlamm im Faulturm kann der Biogasertrag soweit erhöht werden, dass eine Energieautarkie an der ARA erreicht und elektrische Energie oder aufbereitetes Methangas in das öffentliche Netz eingespeist werden kann.

Wegen hohen Anteilen an anaerob leicht abbaubaren und energetisch hochwertigen Substanzen sind Reststoffe aus der Lebensmittelindustrie, wie Molkerei-, Schlachterei- oder Bäckereiabfälle sowie Fettabscheiderinhalte sehr gut geeignete Co-Substrate. Alle diese Substrate zeichnen sich dadurch aus, dass sie im Vergleich zu Siedlungsabfällen eine relativ homogene Zusammensetzung haben und in der Regel nur wenige Störstoffe beinhalten. $\mathrm{Zu}$ Störstoffen sind sämtliche nicht biologisch abbaubare Feststoffe zu zählen, die in den Aggregaten zu Problemen führen können. Die Störstoffe können in folgende vier Bereiche hinsichtlich Schadenspotenzial gegliedert werden:

1. Blockaden,

2. Verschleiß,

3. Schwimmschichten,

4. Sinkschichten.

Längliche Kunststofffolien oder Textilien können sich um bewegte Teile wie Absperrarmaturen oder Rührwerke wickeln und durch diese Zopfbildung die Aggregate einerseits zum Blockieren bringen oder - im Fall der Rührwerke - zum Bruch oder Abreißen führen, wenn die Belastung durch das Gewicht der Zöpfe zu groß wird. Ebenso können diese leichten Störstoffe Schwimmschichten bilden. Inerte oder schwere Partikel im Faulschlamm wie Steine oder Glas verursachen einen höheren Verschleiß der Rohrleitungen und an Anlagenteilen wie Pumpen oder den Entwässerungseinrichtungen. $\mathrm{Zu}$ sätzlich setzen sie sich am Boden des Faulturms ab, wodurch zum einen das Nutzvolumen sinkt und zum anderen Rührwerke beschädigt werden können, sobald diese in die Sinkschicht hineinragen.

Seit einigen Jahren werden neben den bereits angeführten störstoffarmen 


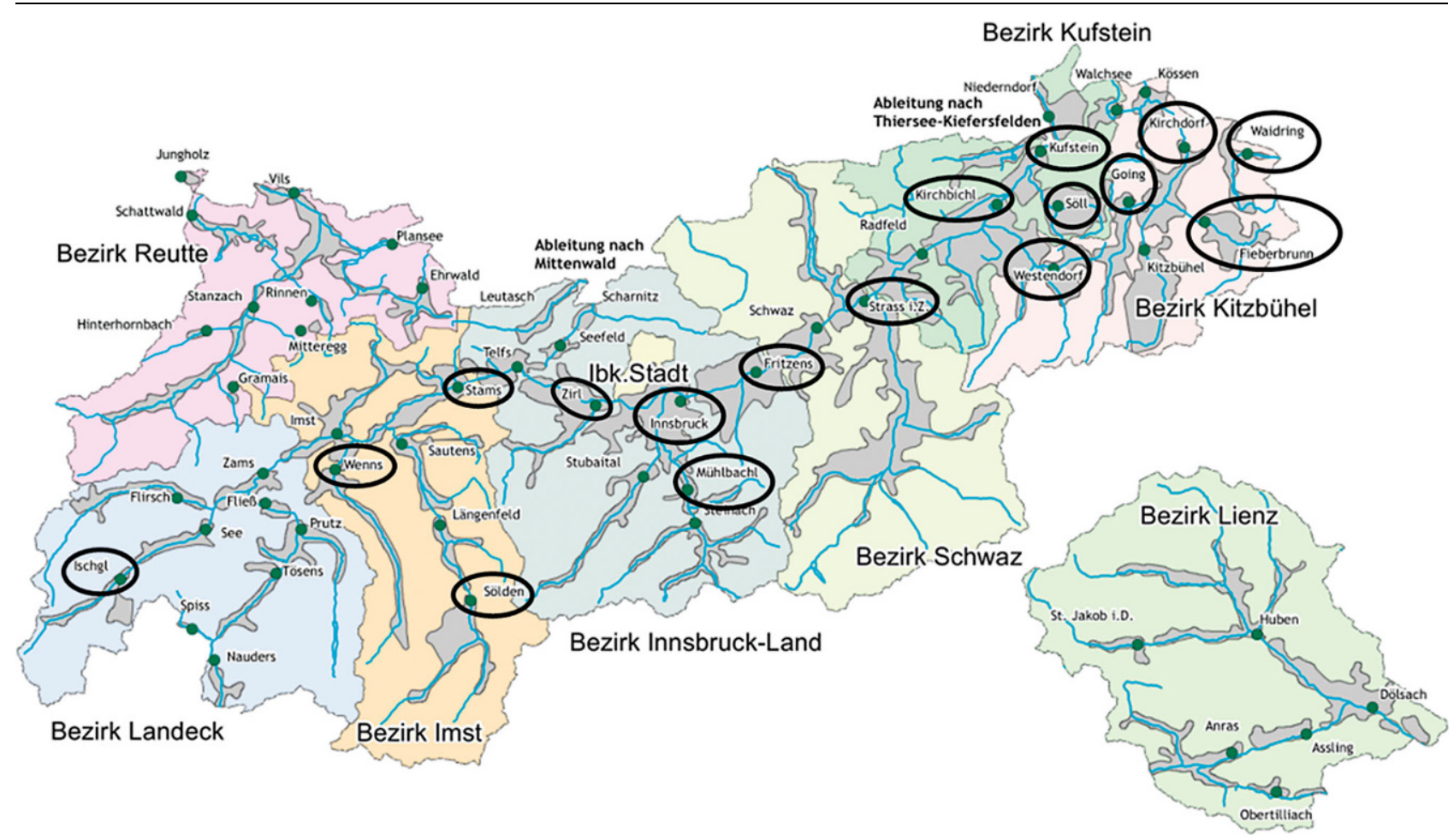

Abb. 1 Tiroler Kläranlagen mit Co-Vergärung (Meirer et al. 2016a; nach Land Tirol 2014; Mölgg 2016 [Persönliche Mitteilung per E-Mail am 18.07.2016.])

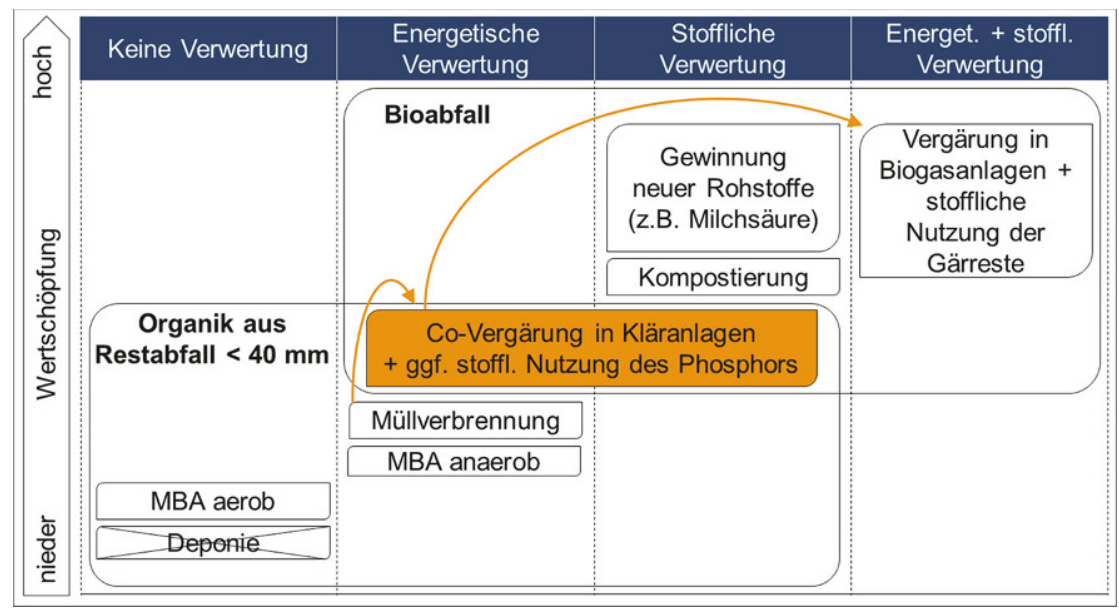

Abb. 2 Verwertungswege von Bioabfall bzw. der NK aus Restabfall nach Meirer et al. (2016b)

Co-Substraten auch getrennt gesammelte Bioabfälle in Faultürmen von Kläranlagen eingesetzt. Da diese nicht nur mineralische Anteile, z.B. durch mit Topfpflanzen eingebrachter Erde, sondern auch Fehlwürfe wie Metallteile oder Ähnliches beinhalten, müssen diese Abfälle vor einer Verwendung als Co-Substrat aufbereitet werden.

In Österreich ist der Trend zur CoVergärung vor allem in Tirol zu erkennen. Im Jahr 2011 wurden in 13 Klär- anlagen 18.130 Mg/a an Co-Substraten mitvergoren (Prantauer 2013). Nach Ebner und Pümpel (2016) ist diese Menge in den darauffolgenden fünf Jahren auf etwa $25.000 \mathrm{Mg} / \mathrm{a}$ angestiegen, welche nach Mölgg (Persönliche Mitteilung per E-Mail am 18.07.2016.) in 17 CoVergärungsanlagen eingesetzt wurden (Abb. 1).

Die genannten $25.000 \mathrm{Mg} / \mathrm{a}$ setzen sich aus ansteigenden Mengen an getrennt gesammeltem Bioabfall zusammen, wovon derzeit in Tirol $51.200 \mathrm{Mg} / \mathrm{a}$ anfallen (BMLFUW 2017). Diese Entwicklung führt zu einem zunehmenden Wettbewerb um diese biogenen Abfälle, die bisher hauptsächlich in Kompostierungs- und Biogasanlagen verwertet wurden und damit auch in Konkurrenz zur stofflichen Verwertung vom erzeugten Produkt Kompost stehen.

\subsection{Substitut für etablierte Co- Substrate}

In Abb. 2 sind die einzelnen Verwertungsmöglichkeiten von Bio- und Restabfall bzw. der Organik aus Restabfall $<40 \mathrm{~mm}$ dargestellt. Eine Monovergärung biogener Abfälle und anschließende Kompostierung wäre demnach erstrebenswert, gefolgt von einer stofflichen Nutzung. Die gesetzliche Lage in drei ${ }^{1}$ und bald allen neun Bundesländern Österreichs verbietet eine Ausbringung der getrennt gesammelten Bioabfälle nach einer Co-Vergärung mit

${ }^{1}$ In Tirol, Wien und größten Teilen Salzburgs besteht dieses Verbot in den Landesgesetzbüchern bereits (LGBl. (Salzburg) Nr. 80/2001 (2002); LGBl. (Tirol) Nr. 130/2013, (2013); LGBl. (Vienna) Nr. 08/ 2000, (2015)). 
Tab. 1 Sortierkatalog unter Berücksichtigung der Korngröße

\begin{tabular}{|c|c|c|c|}
\hline \multicolumn{4}{|l|}{$\begin{array}{l}\text { Restabfall } \\
\text { Niederkalorik }\end{array}$} \\
\hline $40-250 \mathrm{~mm}$ & $10-40 \mathrm{~mm}$ & $2-10 \mathrm{~mm}$ & $0-2 \mathrm{~mm}$ \\
\hline \multirow[t]{4}{*}{-} & $10-16 \mathrm{~mm}$ & $2-4 \mathrm{~mm}$ & - \\
\hline & $16-25 \mathrm{~mm}$ & $4-6,3 \mathrm{~mm}$ & \\
\hline & $25-31,5 \mathrm{~mm}$ & $6,3-10 \mathrm{~mm}$ & \\
\hline & $34,5-40 \mathrm{~mm}$ & - & \\
\hline Organik & Organik & Organik & - \\
\hline Kunststoff 2D & Kunststoff 2D & \multirow[t]{2}{*}{ Kunststoff } & - \\
\hline Kunststoff 3D & Kunststoff 3D & & - \\
\hline Steine \& Keramik & Steine/Keramik & Steine/Keramik & Inertstoffe \& Asche \\
\hline Glas & Glas & Glas & Glas \\
\hline Metall Fe & Metall Fe & \multirow[t]{2}{*}{ Metall } & \multirow[t]{2}{*}{ Metall } \\
\hline Metall NE & Metall NE & & \\
\hline Papier & Papier & - & - \\
\hline Textil & Textil & - & - \\
\hline Holz & Holz & - & - \\
\hline Problemstoffe & Problemstoffe & - & - \\
\hline Rest & Rest & Rest & Rest \\
\hline Verbundstoffe & Verbundstoffe & - & - \\
\hline$<40 \mathrm{~mm}$ & - & - & - \\
\hline- & - & - & Glühverlust \\
\hline
\end{tabular}

Klärschlämmen. Somit wird eine stoffliche Verwertung nahezu ausgeschlossen und einzig eine Phosphorrückgewinnung nach der Mono-Verbrennung zukünftig ermöglicht. Dafür gibt es bereits zahlreiche Technologien, die technisch ausgereift, jedoch erst vereinzelt angewendet werden (Egle 2016).

Für Restabfall hat sich die Verbrennung seit einigen Jahren als Behandlungsmethode durchgesetzt, womit das Recycling bis auf einige wenige Metalle beschränkt wird. In der Folge gehen biogene Abfälle verloren, die $\mathrm{zu}$ ca. $18 \%^{2}$ im Restabfall enthalten sind (BMLFUW 2017).

Würden die im Restabfall enthaltenen biogenen Reststoffe mechanisch abgetrennt und als Substitut für die derzeit als Co-Substrat verwendeten getrennt gesammelten Bioabfälle genutzt, könnten die derzeit als Co-Substrate eingesetzten getrennt gesammelten Bioabfälle wieder höherwertiger verwertet werden. Als zusätzlicher Nebeneffekt könnte zukünftig auch Phosphor aus Teilen des Restabfalls rückgewonnen werden. Die Phosphorrückgewin-

${ }^{2}$ Der tatsächliche Anteil an biogenen Abfällen liegt noch ca. $5 \%$ höher, da ca. $9 \%$ an Feinfraktion kleiner $20 \mathrm{~mm}$ festgestellt wurden (BMLFUW 2017). Diese Feinfraktion besteht aus großteils biogenen Abfällen, wie eigene Analysen in Abschn. 3.1 zeigen. nung aus Klärschlämmen ist aufgrund der höheren Phosphor-Konzentrationen und der homogeneren Zusammensetzung der Rückstände aus der MonoVerbrennung einfacher als bei Verbrennungsanlagen für gemischte Siedlungs-, Sperr- und weitere Abfälle, bei denen der Phosphor nach Egle et al. (2016) als verloren betrachtet werden muss.

\section{Methoden}

Um die Möglichkeit der mechanischen Abtrennung biogener Abfälle aus Restabfällen untersuchen zu können, wurden Restabfälle vorab über einen Zeitraum von zweieinhalb Jahren regelmärien charakterisiert. Anschließend wurden darauf abgestimmte Aufbereitungsmethoden ausgewählt und in neun Versuchsreihen auf ihre Eignung für die vorliegende Aufgabenstellung untersucht. Ebenso wurden die je nach Aufbereitungsmethode im Substrat noch enthaltenen Störstoffe bestimmt.

\subsection{Restabfallcharakterisierungen}

Die Proben wurden in der mechanischen Abfallbehandlungsanlage (MA) Ahrental gezogen. Dort wird der Restabfall der Bezirke Innsbruck Stadt, -Land sowie Schwaz zerkleinert und bei $40 \mathrm{~mm}$ gesiebt. Da aus Literaturangaben sowie aus eigenen Analysen ßig beprobt und nach mehreren Krite- bekannt ist, dass die Feinfraktion einen deutlich höheren Anteil an biogenen Abfällen enthält, wurde in weiterer Folge nur der Restabfall $<40 \mathrm{~mm}$ untersucht.

Dieser wurde zunächst in acht Korngrößenklassen (KGK) gesiebt, welche anschließend einzeln sortiert wurden (siehe Tab. 1).

Die vier KGK zwischen 10 und $40 \mathrm{~mm}$ wurden in 13 verschiedene Fraktionen sortiert. Bei Kunststoffen wurde in Folien (2D) und rollende Kunststoffe (3D), bei den Metallen in eisen- und nichteisenhaltige Teile unterschieden, da diese physikalischen Eigenschaften für die spätere Aufbereitung wichtig waren. Bei den drei KGK zwischen 10 und $2 \mathrm{~mm}$ wurde eine Vereinfachung auf sechs Fraktionen vorgenommen. Die Fraktion kleiner $2 \mathrm{~mm}$ wurde zuerst getrocknet und geglüht, bevor die Glührückstände sortiert wurden. Sowohl der Wasserverlust beim Trocknen als auch der Glühverlust wurden bei der Auswertung zur Fraktion „Organik“ gezählt. Ebenso wurde diese Fraktion sowohl im trockenen als auch im geglühten Zustand in sechs weitere KGK gesiebt (vgl. nachfolgende Abbildung). Diese Korngrößenverteilungen liefern wichtige Daten für die Auslegung einer Feinstaufbereitung wie beispielsweise mit einem Hydrozyklon.

Von allen charakterisierten Restabfällen $<40 \mathrm{~mm}$ wurde zusätzlich der Trockenrückstand (TR) bzw. organische TR (oTR) bestimmt. Ebenso wurde in regelmäßigen Abständen das Biogaspotenzial ermittelt. Ein Überblick über die durchgeführten Charakterisierungen ist in Abb. 3 ersichtlich.

\subsection{Mechanische Abtrennung biogener Abfälle aus Restabfall}

Für die Abtrennung der biogenen Abfälle aus dem Restabfall $<40 \mathrm{~mm}$ wurden eine Reihe von Aufbereitungsverfahren ausgewählt, die wie folgt unterteilt wurden:

1. Trockene Abfallaufbereitung,

2. optional trockene oder nasse Abfallaufbereitung,

3. nasse Abfallaufbereitung.

Bei den Charakterisierungen der aufbereiteten Proben wurden, sofern möglich, dieselben Analysen wie bei den Restabfällen $<40 \mathrm{~mm}$ durchgeführt. Die Sortierungen wurden jedoch großteils durch andere Methoden ersetzt, da die durch die Aufbereitung zum Teil zu Ag- 


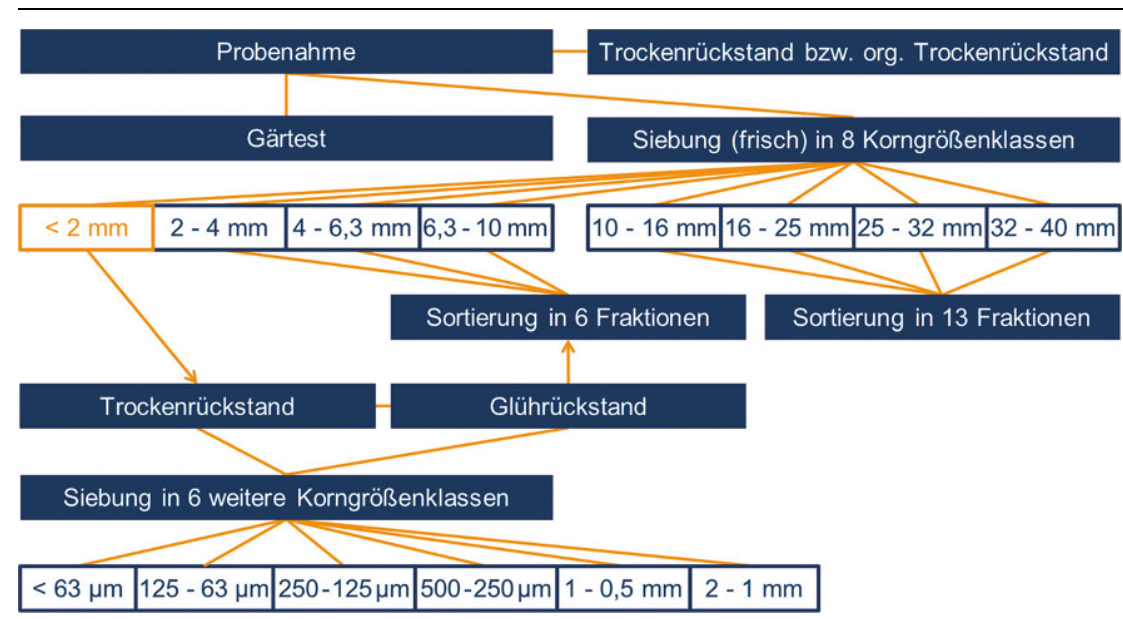

Abb. 3 Methodik der Restabfallcharakterisierungen nach Meirer et al. (2016b)

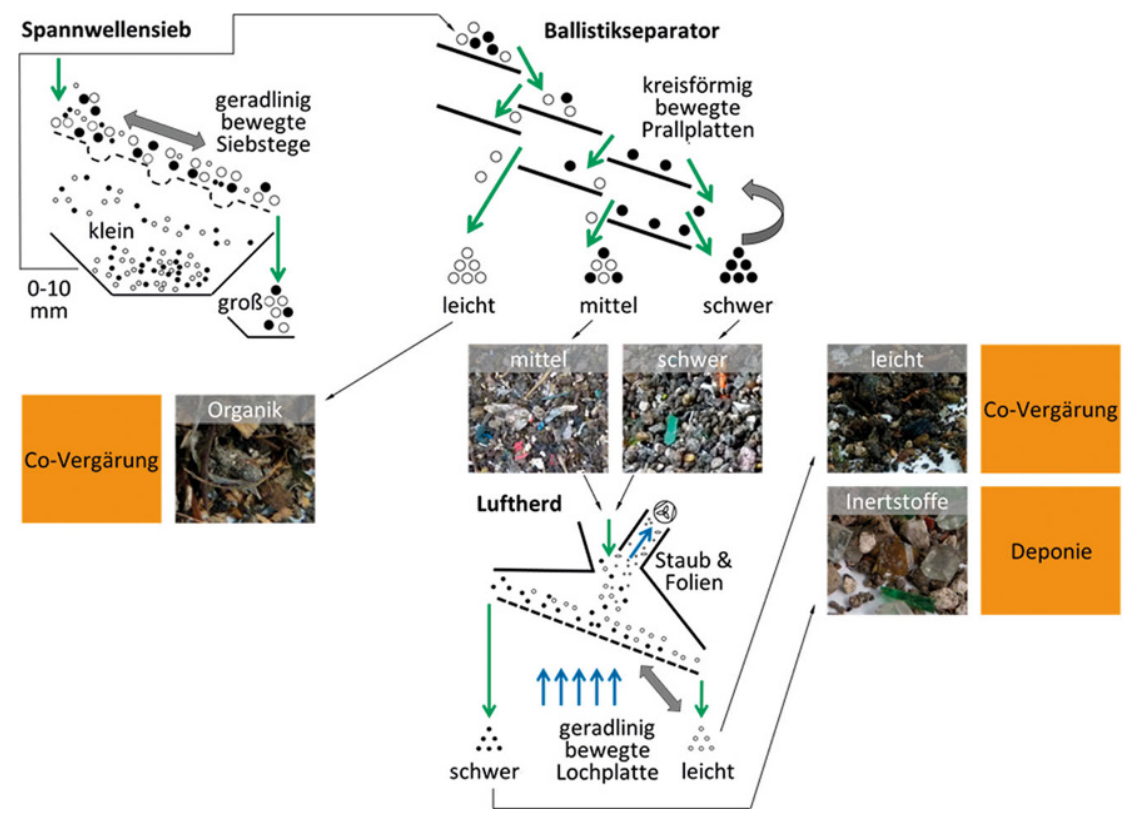

Abb. 4 Schema trockene Aufbereitung nach Meirer et al. (2017a)

glomeraten verpressten Abfälle kaum sortierbar waren. Bei manchen Versuchsreihen wurden sowohl der Input als auch die einzelnen aufbereiteten Fraktionen analysiert oder die Zusammensetzung des ursprünglichen Abfalls aus den aufbereiteten Fraktionen errechnet. Der Anteil biologisch abbaubarer Stoffe im aufbereiteten Substrat wurde mit Hilfe des oTR-Gehalts und des Biogaspotenzials bestimmt. Die im Substrat enthaltenen Störstoffe wurden durch Siebungen unter laufender
Wasserzugabe $^{3}$, im getrockneten als auch im geglühten Zustand, ermittelt.

Da bei allen Versuchsreihen unterschiedliche Abfälle verwendet wurden, können die verschiedenen Aufbereitungsverfahren nur bedingt miteinander verglichen werden. Großteils wurden die Restabfälle von der MA Ahrental (wie auch für die Charakterisierungen vgl. 2.1), aufgrund der Anla-

3 Diese Methode erwies sich bei den mittels Abfallpressen aufbereiteten Proben als sehr hilfreich, um die Agglomerate aufzulösen, ohne die Korngröße der einzelnen Partikel durch Brechen oder Reißen zu verändern. Die einzelnen Siebrückstände wurden nach dem nassen Sieben getrocknet, bevor sie gewogen wurden. genstandorte teilweise aber auch Abfälle aus Deutschland oder Norwegen verwendet. ${ }^{4}$

\subsubsection{Trockene Aufbereitung}

Bei der trockenen Aufbereitung wurden drei Versuchsreihen mit drei Anlagen in zwei unterschiedlichen Anordnungen durchgeführt. Abb. 4 skizziert das Fließbild einer Kombination von Spannwellensieb, Ballistikseparator und Luftherd. Der Siebdurchgang $<10 \mathrm{~mm}$ vom Spannwellensieb wurde am Ballistikseparator durch kreisförmig bewegte Platten in eine organikreiche Leicht-, Mittel- bzw. Schwerfraktion aufbereitet. Letztere wurden am Luftherd durch eine perforierte Platte fluidisiert und in eine weitere organikreiche Leicht- und eine Schwerfraktion getrennt.

In einer zweiten Anordnungsmöglichkeit wurden die Siebdurchgänge $<5 \mathrm{~mm}$ sowie $<10 \mathrm{~mm}$ nach dem Spannwellensieb direkt auf den Luftherd aufgegeben. Durch den im Vergleich zum Ballistikseparator begrenzten Durchsatz des Luftherds müssten bei dieser Anordnung jedoch doppelt so viele Luftherde verwendet werden, wie bei der in Abb. 4 skizzierten Abfolge, um dieselbe Abfallmenge behandeln $\mathrm{zu}$ können.

\subsubsection{Optional trockene oder nasse Aufbereitung}

Bei der zweiten Kategorie an Aufbereitungsanlagen, die optional ohne oder mit Zugabe von Wasser betrieben werden können, wurden drei verschiedene Kolben- und eine Schneckenpresse untersucht.

Bei den Kolbenpressen wird der Abfall mittels Presskolben (Abb. 5), bei der Schneckenpresse mittels Schnecke gegen eine Pressmatrize gepresst. Das Feinmaterial bzw. komprimierbare Abfälle wie beispielsweise Lebensmittelabfälle gelangen dadurch in den Pressdurchgang, harte, große Partikel verbleiben im Pressrückstand.

Der Durchmesser der Presslöcher variierte zwischen 5 und $12 \mathrm{~mm}$, der Pressdruck zwischen 3 und knapp 70 bar.

${ }^{4}$ Es konnte bei den Charakterisierungen nicht aufbereiteter Restabfälle im Zeitraum von zweieinhalb Jahren festgestellt werden, dass die Zusammensetzungen von Abfällen derselben Region großen Schwankungen unterliegen. 


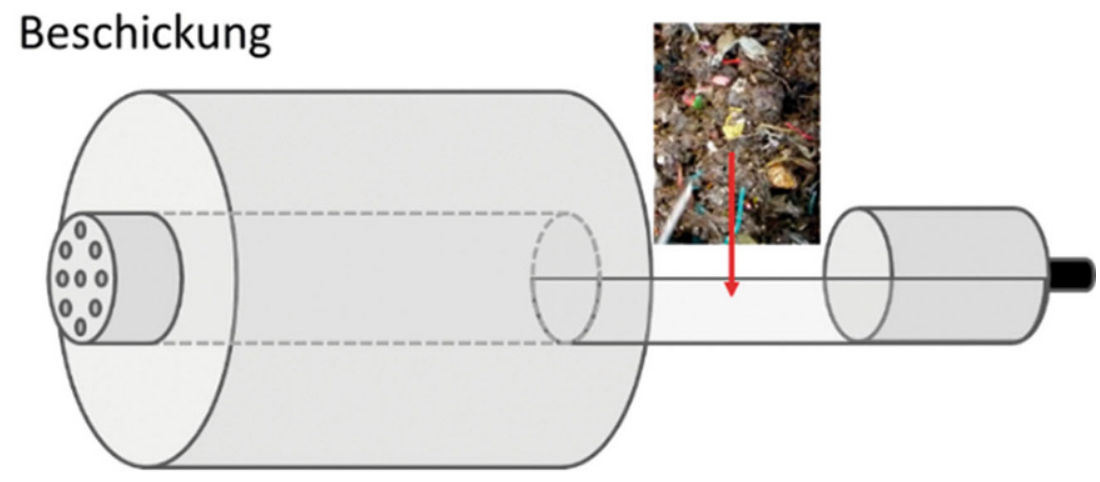

\section{Pressvorgang}

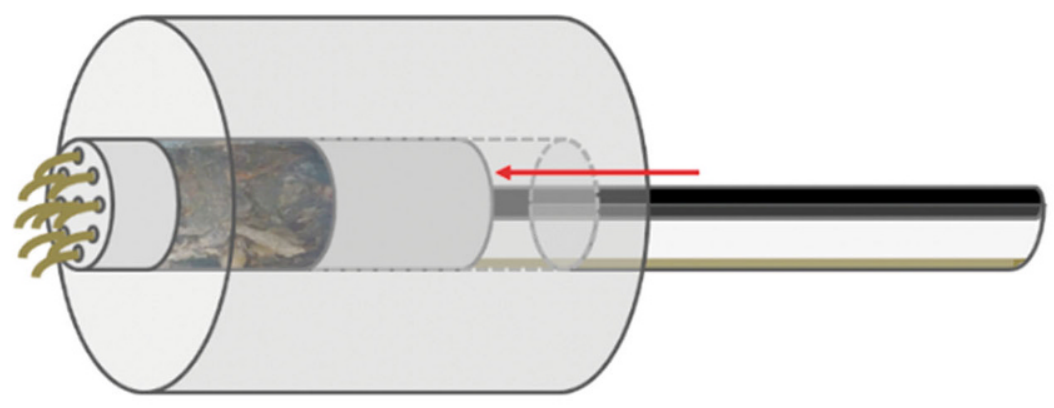

\section{Entleerung}

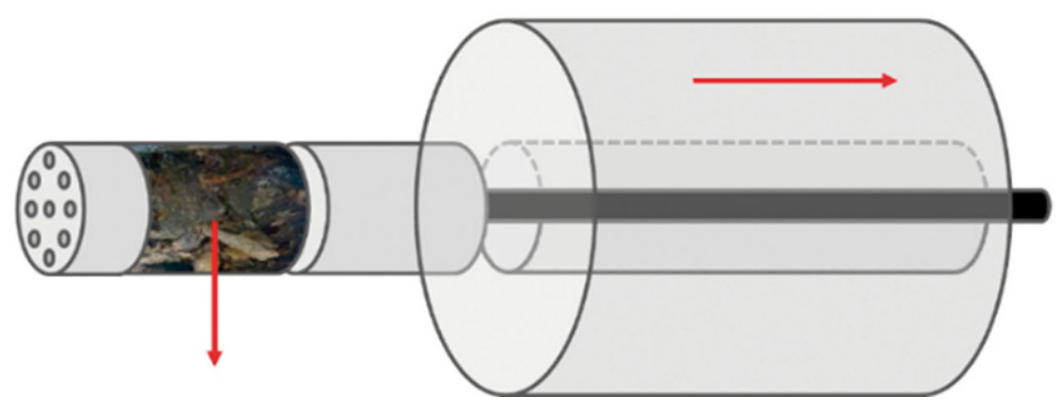

Abb. 5 Schema einer Kolbenpresse nach Meirer et al. (2017a)

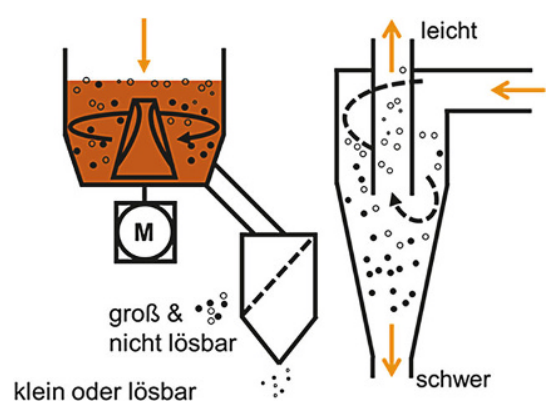

Abb. 6 Schema eines Pulpers + Hydrozyklons (Meirer et al. 2017b)

Density- und Low-Density-Pulpern unterschieden. High-Density-Pulper werden mit einem TR-Gehalt von um die $20 \%$, Low-Density-Pulper mit TR-Gehalten von bis zu unter $10 \%$ betrieben wurde ein High-Density-Pulper getestet, der in einer Biogasanlage zur Aufbereitung getrennt gesammelter Bioabfälle vor einer Nassvergärung eingesetzt wird. ${ }^{5}$

\section{Ergebnisse}

\subsection{Restabfallcharakterisierungen}

Die Zusammensetzung des Restabfalls $<40 \mathrm{~mm}$ der MA Ahrental ist in Abb. 7 dargestellt. Sie stellt den Mittelwert von zwölf Sortierungen dar, die mit Laborproben von jeweils durchschnittlich sechs qualifizierten Stichproben erstellt wurden. In Summe wurden ca. $75 \mathrm{~kg}$, verteilt auf die einzelnen acht KGK, sortiert.

In Abschn. 1.3 wurde für den gesamten Restabfall ein Anteil biogener Abfälle von rund $20 \%$ angegeben. Dieser Wert lag im Restabfall $<40 \mathrm{~mm}$ bei mehr als dem Doppelten. In Abb. 8 sind Verläufe gruppierter Abfallfraktionen in Bezug zur Korngröße dargestellt.

Es ist zu erkennen, dass die Organikgehalte in den KGK bis $10 \mathrm{~mm}$ sehr stark anstiegen, wodurch sich in der Fraktion $<10 \mathrm{~mm}$ bereits ca. $70 \%$ der im gesamten Restabfall $<40 \mathrm{~mm}$ enthaltenen Organik wiederfanden. Die brennbaren Anteile wie Kunststoffe, Papier, Textil und Holz nahmen hingegen erst $\mathrm{ab}>10 \mathrm{~mm} \mathrm{zu}$, wodurch diese Störstoffe bereits durch eine weitere Siebung bei $10 \mathrm{~mm}$ größtenteils abgetrennt werden können. Der Organikgehalt würde durch diese Siebung im Siebdurchgang auf ca. $67 \%$ ansteigen, wobei nur ca. $30 \%$ der Organik mit dem Siebüberlauf verloren gehen würden.

Die Schwerfraktion bestand aus Steinen, Glas und Metallen und ließ einen ähnlichen Verlauf erkennen wie die Organik, weshalb diese Störstoffe ebenfalls in der KGK $<10 \mathrm{~mm}$ vorhanden waren und vor einer Co-Vergärung abgetrennt werden müssen.

Der TR-Gehalt der untersuchten Restabfallproben $<40 \mathrm{~mm}$ betrug im Mittel 63,8 \pm 4,5\%, der oTR-Gehalt $57,9 \pm 6,1 \%$ bezogen auf den TR.

Die Analysen des Biogaspotenzials zeigten eine durchschnittliche Gaspro(Bischofsberger et al. 2005; Ek 2016; Warnke 2016: Persönliche Mitteilung. 06.04.2016). Im vorliegenden Projekt
5 Bei den anderen Versuchsreihen wurde großteils Restabfall $<40 \mathrm{~mm}$ eingesetzt. In einzelnen Versuchen wurde auch Restabfall $<60$ bzw. $<80 \mathrm{~mm}$ oder eine Mischung aus Rest- und Sperrabfall aufbereitet. 


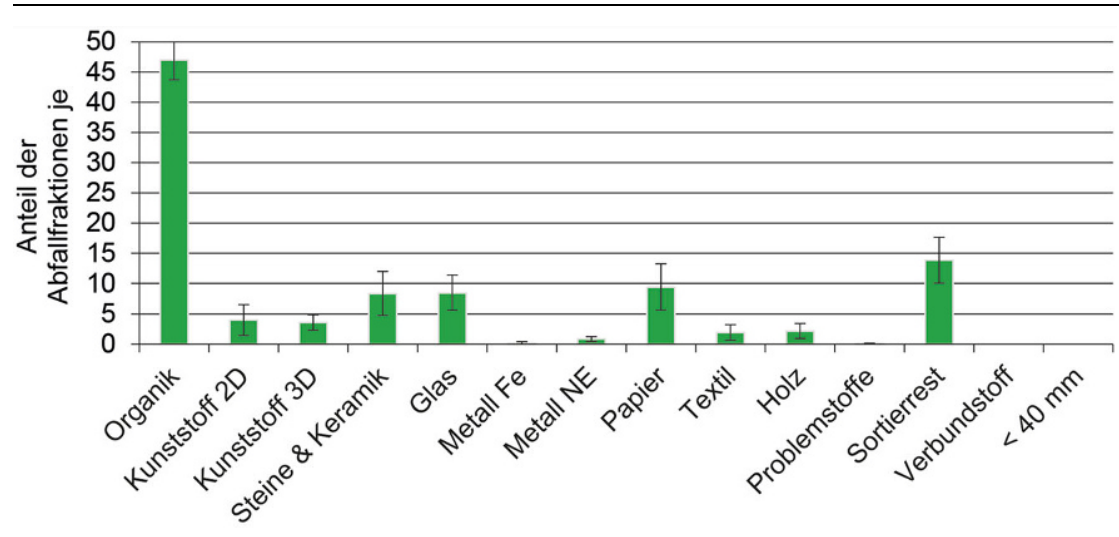

Abb. 7 Zusammensetzung von Restabfall $<40 \mathrm{~mm}$

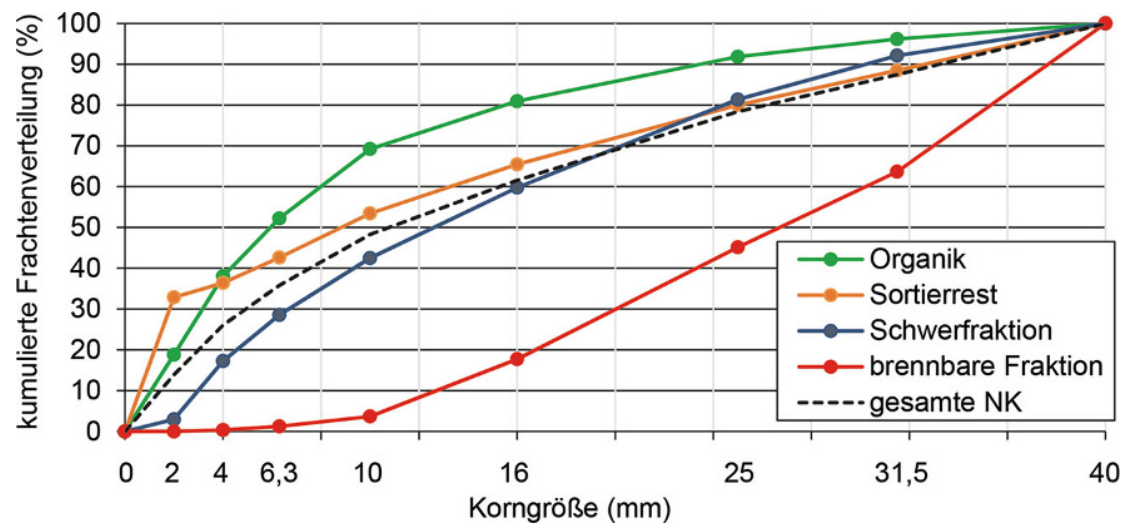

Abb. 8 Kumulierte auf jeweils $100 \%$ bezogene Frachtenverteilung gruppierter Fraktionen bzw. der Korngrößenverteilung des gesamten Restabfalls $<40 \mathrm{~mm}$ nach Meirer et al. (2016b)

duktion des Restabfalls $<40 \mathrm{~mm}$ von $175 \mathrm{~m}^{3}$ Biogas (BG) pro Mg Frischmasse bzw. $469 \mathrm{~m}^{3}$ BG/Mg oTR.

\subsection{Mechanische Abtrennung biogener Abfälle aus Restabfall}

\subsubsection{Trockene Aufbereitung}

Bei den Versuchsreihen zur trockenmechanischen Aufbereitung konnten je nach Anlagenkombination und Siebmaschenweite nur 19 bis $29 \%$ des im Restabfall $<40 \mathrm{~mm}$ enthaltenen TR bzw. 25 bis $30 \%$ des oTR in das potenzielle Substrat gebracht werden. Hauptgrund für diese geringen Transferraten im Vergleich zu den nachfolgenden Aufbereitungsversuchen war die weitere Siebung bei 5 bzw. $10 \mathrm{~mm}$. Der relative Anteil an oTR bezogen auf den TR konnte durch die Aufbereitung jedoch von ca. $60 \%$ auf ca. $80 \%$ gesteigert werden. Damit wurde eine mit Bioabfall aus der getrennten Sammlung vergleichbare Zusammensetzung erreicht. Jank et al. (2015) stellten bei
$25 \%$ des vor der Aufbereitung vorhandenen Biogaspotenzials in das Substrat überführt werden.

\subsubsection{Optional trockene oder nasse Aufbereitung}

Die Versuche wurden teilweise mit frischem, teilweise mit angemaischtem Abfall durchgeführt. Im Vergleich zur trockenmechanischen Aufbereitung konnten bei den Kolbenpressen mit 22 bis $47 \%$ beim TR und 24 bis $54 \%$ beim oTR im Mittel deutlich höhere Transferraten ins potenzielle Co-Substrat den Pressdurchgang - erreicht werden. Bei der Schneckenpresse lagen diese Ergebnisse mit 12 bzw. $17 \%$ hingegen unter jenen der trockenmechanischen Aufbereitung.

Von den inerten Störstoffen wurden bei der Schneckenpresse mit nur $7 \%$ am wenigsten der im Ausgangsmaterial vorhandenen Störstoffe ins Substrat überführt, bei den Kolbenpressen waren die Transferraten in das Substrat mit 19-39\% deutlich höher.

Bei den Kolbenpressen konnte je nach Bauart, Betriebsweise und verwendetem Abfall zwischen 23 und $69 \%$ des im Restabfall $<40 \mathrm{~mm}$ enthaltenen Biogaspotenzials in das CoSubstrat überführt werden. Bei der Schneckenpresse lag dieser Wert bedingt durch die niedrigen TR- und oTR-Frachten bei $30 \%$, wobei ein Biogaspotenzial im Pressdurchgang von bis zu $760 \mathrm{~m}^{3} / \mathrm{Mg}$ oTR erreicht werden konnte.

Bei zwei der drei Kolbenpressen und der Schneckenpresse wurde zum Anmaischen der Abfälle vor dem Abpressen ca. $10 \%$ Wasser, bei einer Kolbenpresse hingegen ca. $60 \%$ zugegeben. Bei letzterer wurden unterschiedlich lange Anmaischdauern und die Einwirkung eines weiteren Pressvorgangs mit dem Pressrückstand der 1. Pressung untersucht.

In beiden Fällen wurde sowohl für den oTR als auch für das Biogaspotenzial ein positiver Effekt auf die Transferraten in den Pressdurchgang beobachtet.

Ein zweites Pressen hatte eine Erhöhung der absoluten oTR-Fracht bzw. des Biogaspotenzials zur Folge, jedoch wurden auch zusätzliche Störstoffe in das Co-Substrat verfrachtet. Der relative oTR-Gehalt bzw. das Biogaspotenzial bezogen auf den oTR-Gehalt blieben dadurch in der Summe der Pressdurchgänge der ersten und zweiten Pressung nahezu unverändert. 


\begin{tabular}{|c|c|c|c|c|c|}
\hline Versuchsanlage & TR & OTR & $\begin{array}{l}\text { Inerte } \\
\text { Störst. }\end{array}$ & Biogas & $\begin{array}{l}\text { Benötigtes } \\
\text { Wasser }\end{array}$ \\
\hline Einheiten & \multicolumn{4}{|c|}{$\%^{*}$} & $\%^{* *}$ \\
\hline Spannwellensieb $5 \mathrm{~mm}+$ Luftherd & 19 & 25 & 10 & 22 & 0 \\
\hline Spannwellensieb $10 \mathrm{~mm}+$ Luftherd & 26 & 30 & 19 & 25 & 0 \\
\hline $\begin{array}{l}\text { Spannwellensieb + Ballistikseparator } \\
+ \text { Luftherd }\end{array}$ & 29 & 26 & 33 & 14 & 0 \\
\hline Kolbenpresse I & 47 & 54 & 39 & 69 & 8 \\
\hline Kolbenpresse II & 37 & 36 & 36 & 23 & 11 \\
\hline $\begin{array}{l}\text { Kolbenpresse III } \\
(1,5 \mathrm{~h} \text { anmaischen, } 1 \mathrm{x} \text { pressen })\end{array}$ & 22 & 24 & 19 & 30 & 61 \\
\hline $\begin{array}{l}\text { Kolbenpresse III } \\
\text { (48 } \mathrm{h} \text { anmaischen, } 1 \text { x pressen) }\end{array}$ & 26 & 27 & 23 & 49 & 61 \\
\hline $\begin{array}{l}\text { Kolbenpresse III } \\
(1,5 \mathrm{~h} \text { anmaischen, } 2 \mathrm{x} \text { pressen) }\end{array}$ & 25 & 27 & 22 & 34 & 61 \\
\hline $\begin{array}{l}\text { Kolbenpresse III } \\
\text { (48 h anmaischen, } 2 \text { x pressen) }\end{array}$ & 27 & 28 & 24 & 51 & 61 \\
\hline Schneckenpresse & 12 & 17 & 7 & 30 & 9 \\
\hline Pulper + Hydrozyklon & 48 & 60 & 22 & 61 & 43 \\
\hline
\end{tabular}

Abb. 9 Vergleich der verschiedenen Aufbereitungsmöglichkeiten anhand der Transferraten in das aufbereitete Substrat nach Meirer et al. (2017a)

\subsubsection{Nasse Aufbereitung}

Mit der Aufbereitung durch den Pulper und den Hydrozyklon konnte mit 48 bzw. $60 \%$ von allen neun Versuchsreihen jeweils die größte Fracht an TR bzw. oTR in das Substrat überführt werden. Von den anfangs im getrennt gesammelten Bioabfall enthaltenen inerten Störstoffen konnten bis auf $22 \%$ alle abgetrennt werden.

Beim Biogaspotenzial konnten $61 \%$ des im unaufbereiteten Bioabfall enthaltenen Potenzials erhalten bleiben, wodurch die $43 \%$ Wasser bezogen auf die ursprüngliche Frischmasse, die zum Anmaischen benötigt wurde, der einzige Nachteil dieser Aufbereitungsvariante waren.

Bei der Untersuchung der verschiedenen Outputströme aus dem Pulper zeigte sich, dass vergleichsweise hohe Mengen an Organik in die abgetrennte Störstofffraktion gelangten. Dies betraf vor allem Zitrusfrüchte, Kartoffeln und Äpfel, die weder bei der dem Pulper vorgeschalteten Zerkleinerung, noch im Pulper selbst, in der knappen Stunde Verweilzeit aufgeschlossen wurden. Sie wurden von dem $6 \mathrm{~mm}$-Sieb abgetrennt und mussten mit den Kunststofffolien und anderen Störstoffen entsorgt werden. Eine Sortieranalyse der nach dem Pulper abgetrennten groben Störstoffe ergab $50 \%$ Organik, $42 \%$ Kunststoff 2D, $1 \%$ Kunststoff 3D, $3 \%$ Glas, $2 \%$ Inertstoffe und $2 \%$ Metalle.
Die Rohsuspension $<6 \mathrm{~mm}$ aus dem Pulper wurde anschließend mit einem TR-Gehalt von 17 und einem oTR-Gehalt von $68 \%$ über einen Hydrozyklon geführt. Mit dem Hydrozyklon wurden $90 \%$ der inerten Partikel größer $125 \mu \mathrm{m}$ abgetrennt. Der oTR-Gehalt der abgetrennten Störstofffraktion lag bei lediglich $36 \%$ und bestätigte damit die hohe Trenneffizienz des Hydrozyklons. Der oTR-Gehalt des aufbereiteten Bioabfalls konnte somit von 71 auf $88 \%$ gesteigert werden.

\subsubsection{Vergleich der verschiedenen Aufbereitungsmöglichkeiten}

In Abb. 9 werden die Frachtenverteilungen des TR, oTR, der inerten Störstoffe und des Biogaspotenzials in das Substrat miteinander verglichen. Ebenso ist der auf die ursprüngliche Frischmasse bezogene Anteil an zugegebenem Wassers zum Anmaischen angeführt. Beim TR, oTR und Biogaspotenzial sind hohe Werte bevorzugt und deshalb grün markiert. Bei den inerten Störstoffen und dem benötigten Wasser zum Anmaischen sind hingegen geringe Werte wünschenswert. Jene Werte bzw. Ergebnisse, die für die vorliegende Fragestellung nachteilige Effekte mit sich bringen, sind rot; Werte, die in der Mitte liegen, gelb markiert.

Die trockenmechanische Aufbereitung zeigte durchwegs schlechtere Ergebnisse als die anderen Aufbereitungs- methoden und hatte einzig den Vorteil, dass kein Wasser benötigt wurde. Sollte diese Variante dennoch angewendet werden, würde es sich anbieten, die Aufbereitung an der MA durchzuführen. Müsste im Gegensatz dazu an der MA bei der Abfallaufbereitung Wasser zugegeben werden, müsste einerseits im Vergleich zu einer Aufbereitung auf der ARA Frischwasser verwendet und andererseits das zugegebene Wasser zusätzlich zur ARA transportiert werden.

Bei den Kolbenpressen erzielte die Presse III durchwegs mittelmäßige Ergebnisse und wies einen hohen Wasserverbrauch beim Anmaischen auf. Die Presse I erreichte bis auf den Anteil an inerten Störstoffen im Substrat sehr gute Ergebnisse, die Presse II gute Ergebnisse.

Die Schneckenpresse erzielte betreffend der Inertstoffe bessere Ergebnisse als die Kolbenpressen, hatte jedoch große Verluste an oTR in den Pressrückstand.

Der Pulper verzeichnete trotz seiner Organikverluste im Vergleich mit den anderen Aufbereitungsmethoden durchwegs sehr gute Resultate und hatte lediglich den Nachteil, dass relativ viel Wasser bei der Aufbereitung benötigt wurde. Wie die Abscheideleistung eines Pulpers mit Restabfall $<40 \mathrm{~mm}$ aussehen würde, kann nur abgeschätzt werden und sollte deshalb in einem weiteren Versuch ermittelt werden.

\section{Zusammenfassung und Ausblick}

Die Co-Vergärung von getrennt gesammelten Bioabfällen hat neben den positiven Effekten, wie der Steigerung der Anlagenauslastung auf ARA und der damit verbundenen Steigerung der Wirtschaftlichkeit, auch einige Nachteile. Durch das Verbot der Ausbringung kommunaler Klärschlämme auf landwirtschaftliche Flächen in Tirol, Wien und Salzburg und der kommenden Ausdehnung dieses Verbots auf ganz Österreich, werden Klärschlämme in den nächsten Jahren zunehmend thermisch verwertet. Eine stoffliche Nutzung der in den biogenen Abfällen enthaltenen Nährstoffe würde somit auf das Element Phosphor begrenzt werden, sofern es zukünftig vermehrt aus den Aschen einer Monoverbrennung rückgewonnen wird. Andererseits könnten bei einer mechanischen Abtrennung der im Restabfall enthaltenen Organik und Nutzung als Co-Substrat die in 
den getrennt gesammelten Bioabfällen enthaltenen Nährstoffe dadurch nach einer Mono-Vergärung und/oder Kompostierung wieder landwirtschaftlich verwertet werden.

In einem laufenden Forschungsprojekt wird daher untersucht, ob es möglich ist, Restabfälle $<40 \mathrm{~mm}$ so aufzubereiten, dass sie als Co-Substrate in den Faultürmen von Kläranlagen verwendet werden können. Dafür wurde die organikreiche Feinfraktion aus einer mechanischen Restabfallbehandlungsanlage ausführlich charakterisiert und verschiedene Aufbereitungsverfahren auf deren Eignung für die vorliegende Themenstellung überprüft.

Die Charakterisierungen zeigten, dass durch eine Siebung bei $40 \mathrm{~mm}$ der im Restabfall enthaltene Anteil an biogenen Abfällen von ca. $20 \%$ im gesamten Restabfall auf ca. $47 \%$ gesteigert werden konnte. Bei einer Siebung auf $10 \mathrm{~mm}$ läge der Organikgehalt sogar bei $67 \%$, allerdings bei geringerer Ausbeute.
Durch die unterschiedlichen Aufbereitungsverfahren konnte die Organik weiter aufkonzentriert bzw. konnten die den Prozess störenden Inhaltsstoffe, insbesondere Inert- und Kunststoffe, deutlich reduziert werden. Das Biogaspotenzial im Substrat konnte damit gesteigert werden.

Um aus den durchgeführten Versuchen Empfehlungen für eine großtechnische Umsetzung ableiten zu können, sollten in einer nächsten Phase Wiederholungen der Versuche durchgeführt werden. Damit könnten Schwankungen des heterogenen Restabfalls ausgeglichen und repräsentative Durchsatzleistungen sowie Wartungs- und Instandhaltungskosten der verschiedenen Aggregate für eine Wirtschaftlichkeitsberechnung bestimmt werden.

Open access funding provided by University of Innsbruck and Medical University of Innsbruck.
Danksagung Wir bedanken uns beim Projektpartner AAG für die gute $\mathrm{Zu}$ sammenarbeit. Diese Arbeit wurde zum einen aus dem alpS-Projekt E02 EnerAlp II finanziert. Das Kompetenzzentrum alpS-K1-Projekt wurde im Rahmen von „COMET Competence Centers for Excellent Technologies" durch BMVIT, BMWFW, die Länder Tirol und Vorarlberg gefördert. Das Programm COMET wurde durch die FFG abgewickelt.

Open Access Dieser Artikel wird unter der Creative Commons Namensnennung 4.0 International Lizenz (http:// creativecommons.org/licenses/by/4. $0 /$ deed.de) veröffentlicht, welche die Nutzung, Vervielfältigung, Bearbeitung, Verbreitung und Wiedergabe in jeglichem Medium und Format erlaubt, sofern $\mathrm{Sie}$ den/die ursprünglichen $\mathrm{Au}$ tor(en) und die Quelle ordnungsgemäß nennen, einen Link zur Creative Commons Lizenz beifügen und angeben, ob Änderungen vorgenommen wurden.

\section{Literatur}

Bischofsberger, W., Dichtl, N., Rosenwinkel, K.H., Seyfried, C.F. (2005): Anaerobtechnik, 2nd ed. Springer Berlin Heidelberg.

BMLFUW (2017): Bundes-Abfall-Wirtschaftsplan 2017 Teil 1.

Ebner, C., Pümpel, T. (2016): Mikrobiologie: Faulturm.

Egle, L., Amann, A., Rechberger, H., Zessner, M. 2016. Phosphor: Eine kritische und zugleich unzureichend genutzte Ressource der Abwasserund Abfallwirtschaft - Stand des Wissens und Ausblick für Österreich und Europa. Österreichische Wasser- und Abfallwirtschaftsterreichische Wasser- und Abfallwirtschaftsterreichische Wasser- und Abfallwirtschaft 68, 118-133. doi:10 1007/s00506-016-0295-6

Ek, P. (2016): Persönliches Gespräch über Pulper und Hydrozyklon. Leiter Business Development Cellwood Machinery AB. 16.02.2016.

Jank, A. et al. (2015): Waste Separation Press (WSP): A mechanical pretreatment option for organic waste from source separation. Waste Management, 39, pp. 71-77. Available at: http://linkinghub.elsevier.com/retrieve/pii/ S0956053X15001269.

Land Tirol (2014): Kläranlagenkataster Tirol 2013.

LGBI. (Salzburg) Nr. 80/2001 (2002): Klärschlamm-Bodenschutzverordnung

LGBI. (Tirol) Nr. 130/2013 (2013): Tiroler Feldschutzgesetz 2000.

LGBI. (Vienna) Nr. 08/2000 (2015): Verbot der Ausbringung von Klärschlamm 2015.

Meirer, M., Müller, W., Bockreis, A. (2016a): Bioabfall vs. organikreiche Restabfälle als Co-Substrat für bestehende Faultürme auf Kläranlagen, in: Recy\&Depotech 2016 Leoben. pp. 335-340

Meirer, M., Müller, W., Bockreis, A. (2016b): Bioabfall vs. organikreichem Restabfall als Co-Substrat für bestehende Faultürme auf Kläranlagen. Präsentation. Recy\&Depotech 2016 Leoben.
Meirer, M., Müller, W., Bockreis, A. (2017a): Innovative Abfallaufbereitung: Erzeugung hochwertiger Substrate für Biogas- \& Kläranlagen. Poster. Österreichische Abfallwirtschaftstagung 2017.

Meirer, M., Müller, W., Bockreis, A. (2017b): Mechanische Aufbereitung organikreicher Restabfälle für die Co-Vergärung in bestehenden Faultürmen auf Kläranlagen, in: 7. Wissenschaftskongress Abfall- \& Ressourcenwirtschaft. Aachen, pp. 129-134

Prantauer, S.T. (2013): Biologische Abfallbehandlung in Tirol - Aktueller Stand und Entwicklungen.

Schneider, I. et al. (2014): Cascading Approach of Biowaste Treatment to Obtain Value-added Organic By-Products and Biogas. In ISWA World Congress Vienna. Wien. 